\title{
AN ALTERNATIVE METRIC FOR CHANNEL ESTIMATION WITH APPLICATIONS IN BLUETOOTH SCHEDULING
}

\author{
João H. Kleinschmidt, Marcelo E. Pellenz and Luiz A. P. Lima Jr.
}

Graduate Program in Computer Science, Pontifical Catholic University of Paraná, CuritibaPR, Brazil.E-mail:\{joaohk, marcelo, laplima\}@ppgia.pucpr.br

\begin{abstract}
Once Wireless Local Networks (WLAN) and Bluetooth devices share the same frequency band (ISM) there is a potential risk of interference if they are supposed to operate close to each other. Additionally, the signal fading effects on mobile Bluetooth networks may deeply affect the overall performance. That is why the use of strategies that minimize transmission on channels with great interference or severe fading is so important. This paper proposes and investigates the use of parameter $m$ of the Nakagami distribution, as the channel estimation metric. We observed that parameter $m$ may provide faster estimates on the channel condition than the bit error rate metric. This metric is applied in a new scheduling algorithm for Bluetooth piconets. Simulation results showing the performance of the algorithm for different traffic conditions are eventually presented.
\end{abstract}

Key words: Bluetooth; wireless networks; Nakagami- $m$ fading; scheduling.

\section{INTRODUCTION}

Bluetooth is emerging as an important standard ${ }^{1}$ for short range and lowpower wireless communications. It operates in the $2.4 \mathrm{GHz}$ ISM (Industrial, Scientific and Medical) band employing a frequency-hopping spread spectrum technique. The transmission rate is up to $1 \mathrm{Mbps}$, using GFSK (Gaussian Frequency Shift Keying) modulation. The Bluetooth MAC protocol is designed to facilitate the construction of ad hoc networks. The devices can communicate with each other forming a network with up to eight nodes, called piconet. Within a piconet, one device is assigned as a master node and the others devices act as slave nodes. Devices in different 
piconets can communicate using a structure called scatternet. The channel is divided in time slots of $625 \mu \mathrm{s}$. A time-division duplex (TDD) scheme is used for full-duplex operation. For data transmission Bluetooth employs seven asynchronous packet types. Each packet may occupy 1, 3 or 5 time slots. The throughput of Bluetooth links using asynchronous packets was investigated ${ }^{2}$ for the additive white Gaussian noise (AWGN) channel and for the Rayleigh fading channel. In other work $^{3}$, we extended the results presented by Valenti ${ }^{2}$ looking into the performance of Bluetooth links in Nakagami- $m$ fading channels.

The sharing of the same frequency band between WLAN and Bluetooth devices may cause interference, if they are operating close to each other. Additionally, may occur mutual interference between different Bluetooth piconets operating in the same area. In Bluetooth networks with node mobility, like in sensor networks applications, the fading effects in the radio signal may significantly decrease the link performance. The use of strategies that minimize the transmission in channels with great interference or severe fading, may substantially improve the piconet performance. Extensive empirical measurements have confirmed the usefulness of the Nakagami- $m$ distribution for modeling radio links ${ }^{13,14}$. The Nakagami- $m$ distribution ${ }^{4}$ allows a better characterization of real channels because it spans, via the parameter $m$, the widest range of multipath fading distributions. For $m=1$ we get the Rayleigh distribution. Using $m<1$ or $m>1$ we obtain fading intensities more and less severe than Rayleigh, respectively.

This work proposes the use of fading parameter $m$ as an alternative channel quality metric. This parameter can be estimated based on the received symbols. In a mobile wireless network, when a node position changes from line-of-sight to non-line-of-sight, for example, the impact in the signal propagation characteristic may be interpreted as a change in the parameter $m$. This model is interesting when Bluetooth devices are applied to ad hoc sensor networks. Power class one Bluetooth devices can cover ranges up to 100 meters, allowing the formation of large area piconets or scatternets. We also propose a new scheduling algorithm for Bluetooth piconets, which uses the channel quality information in the scheduling policy.

This paper is structured as follows: in Section 2 some issues about piconet scheduling and related works are presented. In Section 3 we present and evaluate the performance of the main Nakagami fading parameter estimators found in the literature. Section 4 proposes a new strategy based on channel quality estimation and Section 5 shows the simulation results for different scenarios. Finally, conclusions are drawn in Section 6. 


\section{RELATED WORK ON PICONET SCHEDULING}

In a Bluetooth piconet, the master controls the channel access. A slave can send a packet only if it receives a polling packet from the master. The master transmits packets to the slave in even slots while the slave transmits packets to the master in odd slots. Thus, Bluetooth is a master driven TDD standard and this poses several challenges in scheduling algorithms since there could be a waste of slots if only the master or the slave has data to send. Recently, many schemes have been proposed in the literature for piconet and scatternet scheduling.

In the study of Capone ${ }^{5}$, several polling schemes are compared. In the round robin scheme a fixed cyclic order is defined and a single chance to transmit is given to each master-slave queue pair. The exhaustive round robin (ERR) also uses a fixed order but the master does not switch to the next slave until both the master and the slave queues are empty. The main disadvantage of the ERR is that the channel can be captured by stations generating traffic higher than the system capacity. A limited round robin (LRR) scheme that limits the number $t$ of transmissions can solves this problem. A new scheme called LWRR (limited and weighted round robin) with weights dynamically changed according to the observed queue status is also presented ${ }^{5}$. Other works about piconet scheduling consider QoS issues in Bluetooth ${ }^{6,7}$. The results ${ }^{5,6,7}$ do not consider any loss model for the wireless channels.

In other paper ${ }^{8}$, a scheduling policy based on slave and master queues is shown. The master-slave pairs are distinguished based on the size of the Head-of-the-Line (HOL) packets at the master and slave queues. Then, the pairs are classified in three classes according to slot waste. This information is used in the HOL K-fairness policy (HOL-KFP) ${ }^{8}$. When the authors introduced channel errors, the HOL-KFP had its performance reduced. An extension for HOL-KFP called wireless adapted-KFP (WAKFP) was proposed and the results indicate that a better performance is achieved in the presence of channel errors ${ }^{8}$.

In ${ }^{9}$ an algorithm called Bluetooth Interference Aware Scheduling (BIAS) is presented that uses a channel estimation procedure in order to detect the presence of other wireless devices in the same band (such as other Bluetooth or IEEE $802.11 \mathrm{~b}$ devices). The scheduling algorithm will avoid packet transmission in frequencies that have a high bit error rate (BER), called bad frequencies. This fact reduces the packet loss due to interference of other near devices. Few of the scheduling schemes presented here consider a loss model for the wireless channel. The works ${ }^{8,9}$ use a simple error model. 
This paper models de wireless channel fading through the Nakagami- $m$ distribution, apply an alternative metric for channel state estimation and propose a new scheduling algorithm using that metric. It is important to point out that the proposed metric has a faster estimation convergence than the bit error rate used in ${ }^{8,9}$. The fading parameter $m$ gives us an indication of the fading severity, which will directly impact on either the bit or the packet error rates.

\section{ESTIMATORS FOR THE NAKAGAMI FADING PARAMETER}

The Nakagami probability density function (pdf) is a two-parameter $(m, \Omega)$ distribution, where $m$ is the fading parameter and $\Omega=E\left[r_{n}^{2}\right]$ is the second moment of the received signal samples, $r_{n}$. The estimation of parameter $m$ has found recently many applications, as in systems with optimized transmission diversity. In order to use the Nakagami distribution to model a given set of empirical data, one must determine, or estimate, the fading figure $m$ from the data. Knowledge of the fading parameter is also required by the receiver for optimal reception of signals in Nakagami fading. Many estimators have been proposed in the literature. The fading parameter $m$ is defined as

$$
m=\frac{\Omega^{2}}{E\left[\left(r_{n}^{2}-\Omega\right)^{2}\right]} \quad m \geq 0.5 .
$$

Given $\left\{r_{1}, r_{2}, \ldots, r_{N}\right\}$ as realizations of $N$ i.i.d. Nakagami- $m$ random variates, the $k$ th moment of the Nakagami distribution is given by

$$
\hat{\mu}_{k}=\frac{1}{N} \sum_{i=1}^{N} r_{i}^{k} .
$$

The parameter $m$ may be estimated from its definition, using the 2 nd and 4th sample moments, $\hat{\mu}_{2}$ e $\hat{\mu}_{4}$ :

$$
\hat{m}_{s}=\frac{\hat{\mu}_{2}^{2}}{\hat{\mu}_{4}-\hat{\mu}_{2}^{2}} .
$$

High order sample moments can deviate significantly from the true moments if the sample size is not large enough because outliers. Cheng and Beaulieu ${ }^{11}$ proposed two new estimators, one based in integer moments,

and other based on real moments,

$$
\hat{m}_{t}=\frac{\hat{\mu}_{1} \hat{\mu}_{2}}{2\left(\hat{\mu}_{3}-\hat{\mu}_{1} \hat{\mu}_{2}\right)},
$$




$$
\hat{m}_{1 / p}=\frac{\hat{\mu}_{1 / p} \hat{\mu}_{2}}{2 p\left(\hat{\mu}_{2+1 / p}-\hat{\mu}_{1 / p} \hat{\mu}_{2}\right)}
$$

where $\hat{\mu}_{1 / p}=E\left[r_{i}^{1 / p}\right]$ and $\hat{\mu}_{2+1 / p}=E\left[r_{i}^{2 p+1}\right]$. These estimators are efficient for a moderated number of samples and are appropriated for low complexity implementations. We apply these estimation techniques for a Bluetooth piconet transmission using DM1 packets. For a DM1 packet we have a sequence of $N=240$ channel samples available for channel estimation. These samples represent the soft decision information about the received symbols. We simulate the variability of fading intensity (parameter $m$ ) every new transmission in the same master-slave link of a piconet, during 10 time slots. The simulated and estimated values of parameter $m$ are presented in Table 1 .

Table 1. Simulated and estimated values of parameter $m$

\begin{tabular}{|c|c|c|c|c|c|c|c|c|c|c|c|}
\hline \multicolumn{2}{|l|}{ (2) } & 1 & 2 & 3 & 4 & 5 & 6 & 7 & 8 & 9 & 10 \\
\hline \multirow[t]{2}{*}{$\begin{array}{l}\text { Simulated } \\
\text { value of } m\end{array}$} & & 0.5 & 1 & 0.5 & 1.5 & 1 & 1.5 & 0.5 & 1 & 0.5 & 1.5 \\
\hline & $\hat{m}_{s}$ & 0.60 & 0.93 & 0.80 & 1.74 & 1.27 & 2.17 & 0.56 & 0.99 & 0.50 & 2.22 \\
\hline \multirow{2}{*}{$\begin{array}{l}\text { Estimated } \\
\text { value of } m\end{array}$} & $\hat{m}_{t}$ & 0.63 & 0.95 & 0.94 & 1.87 & 1.35 & 2.32 & 0.63 & 1.07 & 0.48 & 2.33 \\
\hline & $\hat{m}_{\text {1/p }}$ & 0.56 & 0.95 & 0.62 & 1.60 & 1.25 & 2.08 & 0.50 & 0.96 & 0.52 & 2.08 \\
\hline
\end{tabular}

Figure 1 shows the estimators convergence on every time slot of the piconet polling, in a same master-slave link transmission. We may observe that estimator $\hat{m}_{1 / p}$ presents a better convergence. At the end of each time slot transmission we obtain the estimated value, $\hat{m}$. This estimative is applied in the scheduling algorithm proposed in the next section as the channel state information. Notice that tracking the channel condition in one time slot based on the bit error rate may be not feasible due to the low number of available bits from DM1 packet. 


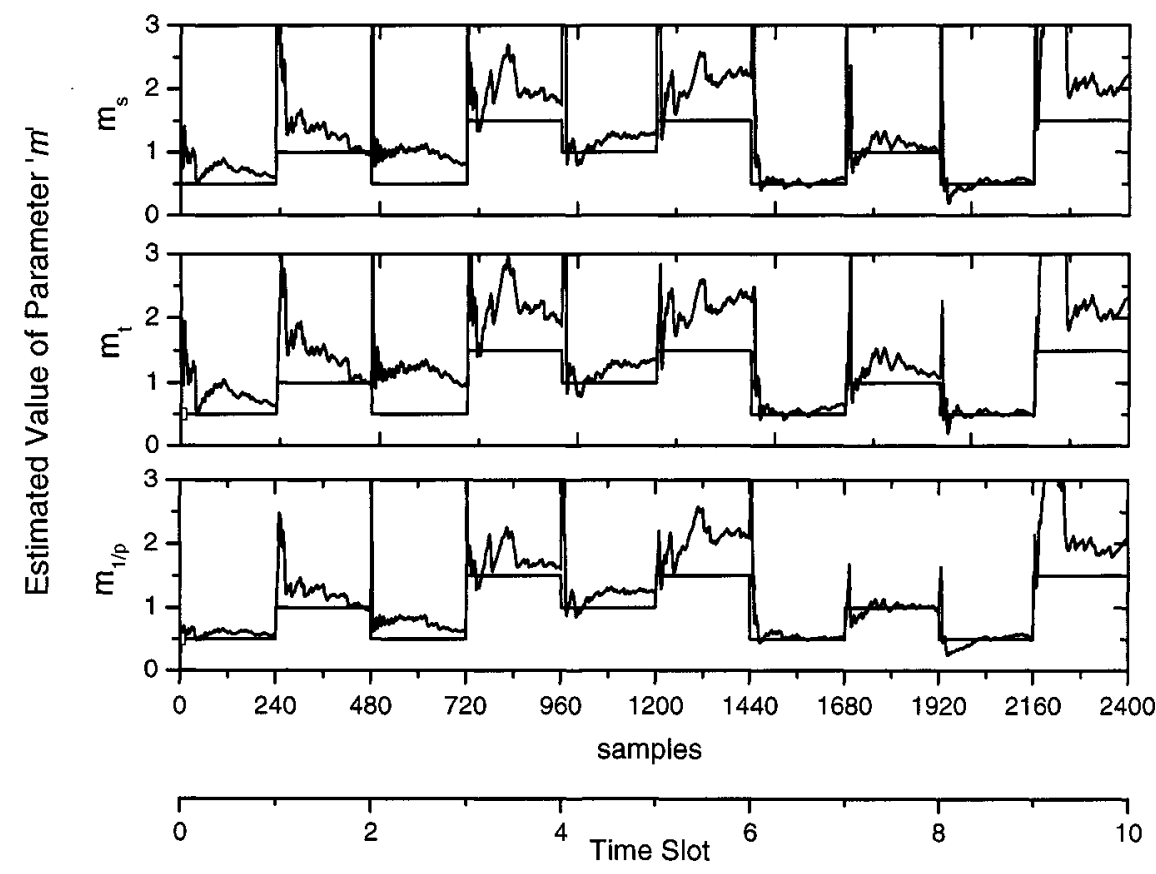

Figure 1. Performance of Nakagami fading parameter estimators in a Bluetooth piconet

\section{PROPOSED SCHEDULING ALGORITHM}

The channel condition can greatly affect the performance of the piconet and the polling strategy. In mobile environments, the status of the wireless channel changes very rapidly and this means that a better performance will be achieved if a node is polled at the moment it has a good channel condition and not polled when the conditions are bad. Since Bluetooth is a technology designed for WPANs (Wireless Personal Area Networks), channels errors due to mobility and interference of other devices are very common. A good scheduling algorithm must consider these issues.

We propose an algorithm - called Bluetooth Channel State Scheduling (BCSS) algorithm - that uses the channel state information for piconet scheduling. The values of the fading parameter $m$ can be efficiently estimated as discussed in Section 3. The master will carry out the estimations using the data packets exchanged with the slaves. Every time a master receives a packet, the value of $m$ for that link will be updated. Since this task does not require extra information to be exchanged between the master and the slaves, no extra time is added to the scheduling policy. In the new 
scheduling policy, the master will poll only the slaves that are above a certain threshold for $m$, indicating a good channel state. The slaves that are below the threshold, indicating that they are at a bad channel state, will be jumped for at most $t_{j}$ times. Notice that if the channel state is always good the algorithm is reduced to a round robin policy.

\section{SIMULATION RESULTS}

We developed an event driven simulator in $\mathrm{C}++$ to compare the BCSS algorithm with round robin and ERR strategies. The effects of Nakagami fading are simulated using the models described in a previous study ${ }^{3}$. A Poisson traffic source was assumed for the traffic generation in each piconet node. This model can simulate various applications of Bluetooth. In the first simulation scenario we investigate the influence of the fading parameter $m$ in a round robin scheduling. It consists of a piconet with a master and 7 slaves separated by a distance $d$. The parameter $\lambda$ is the mean arrival rate in packets per time slot. Fig. 2 shows the average delay for this scenario for three different values of $m$, using DM1 packets. In this scenario all nodes have the same traffic conditions. We can observe that the state of the channel has great influence in the average delay of the piconet, affecting the performance of the network.

In the second simulation scenario a piconet with the master and 4 slaves is considered. Fig. 3 and 4 compare the average delay for different traffic conditions and DM1 packets using round robin, ERR and BCSS algorithm, for distances of seven and ten meters. In the BCSS algorithm we choose $t_{j}=6$ and a threshold $m=1$. This means that only the slaves with $m$ greater than one will be polled, and the others will be jumped for at most six times. The traffic is the same in the master and the slave queues. In the simulation we assume that the channel conditions are changing every two rounds of the polling scheme. We also consider this scenario with $d=10 \mathrm{~m}$ for different traffic conditions in the master and slave links, as defined in Table 2. The results are shown in Fig. 5. The simulation results show that the BCSS algorithm improves its performance when the traffic is high. For low traffic, ERR has the best performance. Other works ${ }^{5,12}$ also concluded that the exhaustive service (ERR) does not have good performance under high traffic. 


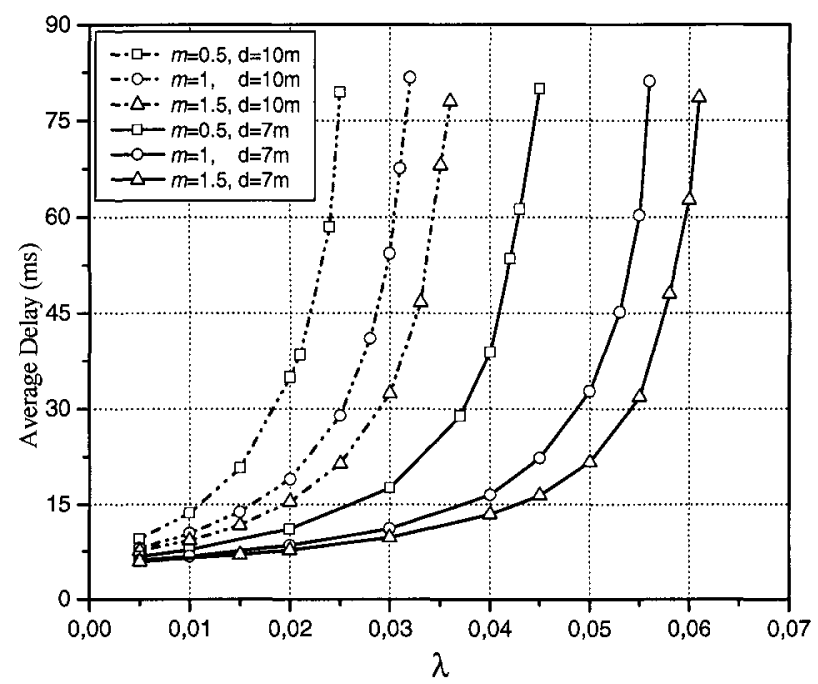

Figure 2. Average delay for different values of $m$

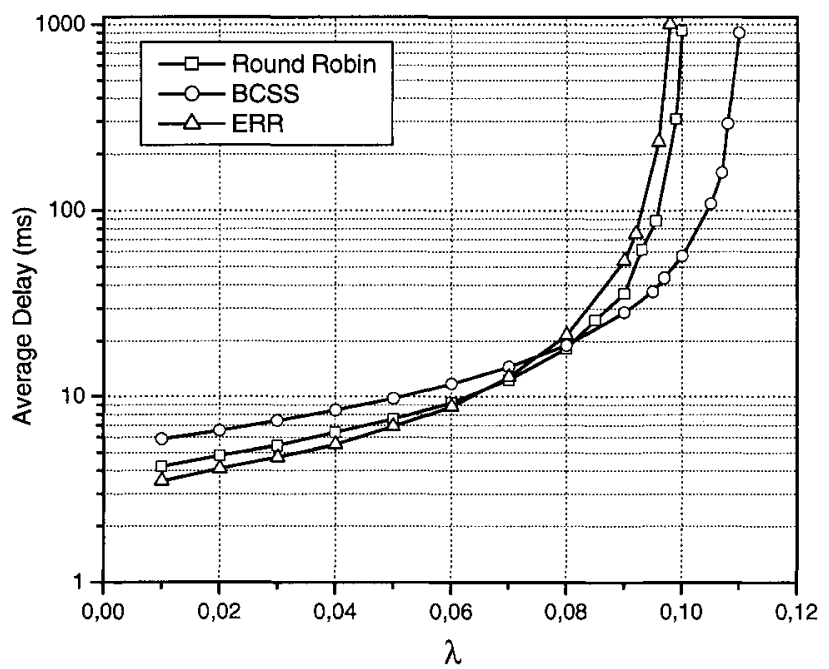

Figure 3. Average delay for $\mathrm{d}=7 \mathrm{~m}$ 


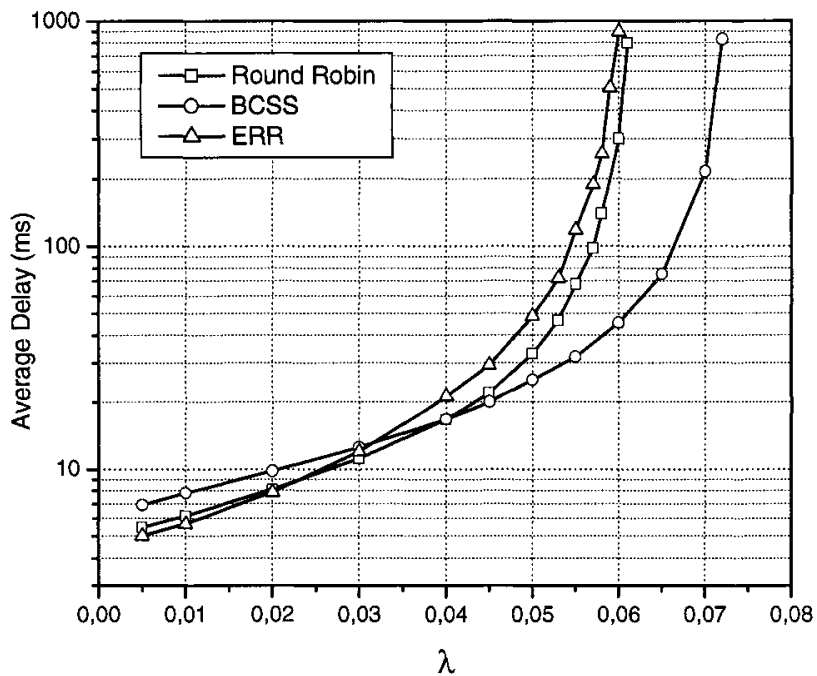

Figure 4. Average delay for $\mathrm{d}=10 \mathrm{~m}$

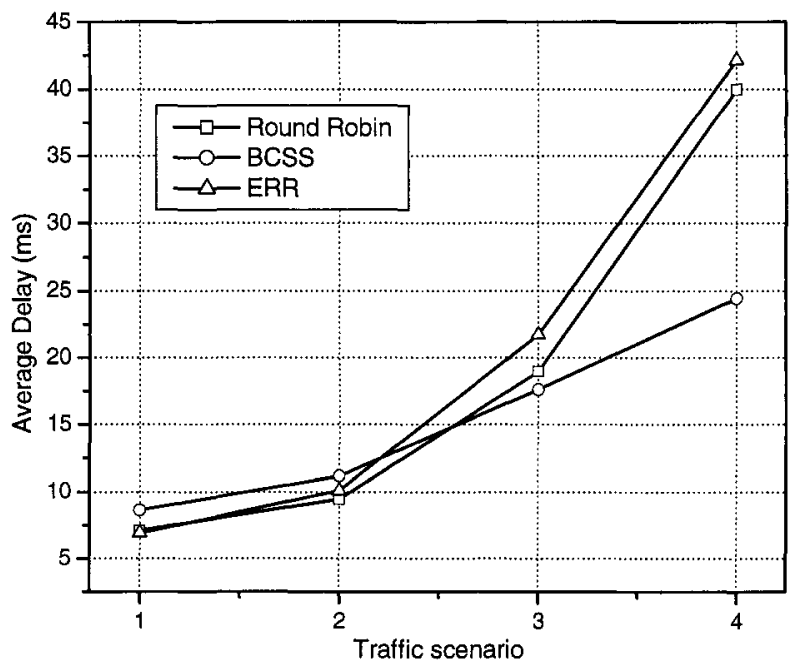

Figure 5. Average delay for different traffic scenarios 
Table 2. Traffic conditions for master-slave links

\begin{tabular}{ccccc}
\hline Traffic scenario & $\begin{array}{c}\lambda_{1} \text { (master- } \\
\text { slave 1) }\end{array}$ & $\begin{array}{c}\lambda_{2} \\
\text { (master-slave 2) }\end{array}$ & $\begin{array}{c}\lambda_{3} \\
\text { (master-slave 3) }\end{array}$ & $\begin{array}{c}\lambda_{4} \\
\text { (master-slave 4) }\end{array}$ \\
\hline 1 & 0.01 & 0.01 & 0.02 & 0.02 \\
2 & 0.01 & 0.01 & 0.04 & 0.04 \\
3 & 0.03 & 0.05 & 0.03 & 0.05 \\
4 & 0.03 & 0.03 & 0.06 & 0.06 \\
\hline
\end{tabular}

\section{CONCLUDING REMARKS}

This paper proposed an alternative metric for channel state estimation using Nakagami- $m$ fading distribution. This metric is applied in the polling strategy of a piconet scheduling algorithm denoted BCSS. The BCSS algorithm is considerably efficient for high traffic loads if the channel conditions change frequently. These variations in channel conditions are present in many applications of the Bluetooth technology in environments with interference and mobility. The BCSS algorithm can be combined with other scheduling policies to improve their performance. This work can be extended to evaluate the performance of the proposed algorithm with different traffic sources, such as FTP, HTTP and voice. Our future works include improvements to the intra-piconet scheduling policy and implementation of an inter-piconet scheduling scheme for scatternets. The parameter $m$ can also be used for other important issues in Bluetooth, like scatternet formation, routing and specific channel coding strategies using AUX1 packets.

\section{REFERENCES}

1. Bluetooth SIG, Specifications of the Bluetooth system, Core Version 1.1, February 2001. http://www.bluetooth.com

2. M. C. Valenti, M. Robert and J.H. Reed, On the throughput of Bluetooth data transmissions, IEEE Wireless Communications and Networking Conference, Orlando, FL, pp. 119-123, March 2002.

3. J. H. Kleinschmidt, M. E. Pellenz, and E. Jamhour, Bluetooth network performance in Nakagami- $m$ fading channels, The Fifth IFIP TC6 International Conference on Mobile and Wireless Communications Networks, Singapore, October 2003.

4. J. Proakis, Digital Communications, New York, NY: McGraw-Hill, $4^{\text {th }}$ edition, 2001.

5. A.Capone, M. Gerla and R. Kapoor, Efficient polling schemes for Bluetooth picocells, IEEE International Conference on Communications, Helsinki, Finland, June 2001.

6. J. B. Lapeyrie and T. Turletti, FPQ: a Fair and Efficient Polling Algorithm with QoS Support for Bluetooth Piconet, IEEE Infocom, San Francisco, CL, 2003. 
7. Y. Liu, Q. Zhang and W. Zhu, A Priority-Based MAC Scheduling Algorithm for Enhancing QoS support in Bluetooth Piconet, IEEE International Conference on Communications, Circuits and Systems, July 2002.

8. M. Kalia, D. Bansal and R. Shorey, MAC Scheduling and SAR Policies for Bluetooth: A Master Driven TDD Pico-Cellular Wireless System, IEEE International Workshop on Mobile Multimedia Communications, San Diego, California, November 1999.

9. N. Golmie, Performance evaluation of a Bluetooth channel estimation algorithm, IEEE International Symposium on Personal Indoor and Mobile Radio Communications, Lisbon, Portugal, September 2002.

10. A. Ramesh, A. Chockalingam and L.B. Milstein, SNR estimation in generalized fading channels and its application to turbo decoding, IEEE International Conference on Communications, Helsinki, Finland, June 2001.

11. J. Cheng and N. C. Beaulieu, Generalized moment estimators for the Nakagami fading parameter, IEEE Communication Letters, vol. 6, no. 4, pp. 144-146, April 2002.

12. N. Johansson, U. Körner and P. Johansson, Performance Evaluation of Scheduling Algorithms for Bluetooth, IFIP TC6 International Conference on Broadband Communications, Hong Kong, November 1999.

13. M. Nakagami, The m-distribution, a general formula of intensity distribution of rapid fading, Statistical Methods in Radio Wave Propagation, W. G. Hoffman, Ed. Oxford, England: Pergamon, 1960.

14. H. Suzuki, A statistical model for urban radio channel model, IEEE Trans. on Communications, vol. 25, pp. 673-680, July 1977. 